\title{
Analysis of hydrogen desorption from linear heating experiments: accuracy of activation energy determinations
}

\author{
M.J. Starink \\ Materials Research Group, Faculty of Engineering and the Environment, University of \\ Southampton, Southampton S017 1BJ, UK, email: m.j.starink@soton.ac.uk
}

\begin{abstract}
Through performing hydrogen desorption experiments at different heating rates, $\beta$, the (effective) activation energy, $E$, of the desorption process can be determined from the shift of a characteristic temperature, $T_{\mathrm{f}}$, of the hydrogen evolution effect with heating rate. In the literature various methods have been employed, and in the present work the accuracy of these methods is investigated. The Kissinger-Akahira-Sunose, Flynn-Wall-Ozawa, Starink, Kissinger and Choo-Lee methods all employ approximations which cause deviations in the activation energy determination, which increase drastically as $E / R T$ ( $R$ is the gas constant) becomes smaller. It is shown that for various hydrogen desorption reactions reported in the literature, deviations in reported $E$ between $\sim 1$ to $\sim 20 \%$ can occur due to inappropriate use of methods. It is shown that the Ozawa and Flynn-Wall-Ozawa methods are highly inaccurate and particularly for hydrogen evolution, where $E / R T$ is often smaller than 15 , they are in most cases inappropriate. The Kissinger peak method is accurate for first order reactions, but as hydrogen evolution reactions generally are not first order reactions, application of this method will result in inaccuracies which increase for decreasing $E / R T$. In general the magnitude of the deviations of such a peak method are not predictable, as this depends on the reaction mechanism. In many cases the Kissinger peak method is inappropriate for high accuracy determination of activation energy for hydrogen evolution reactions. Amongst the methods that provide an activation energy directly from a slope (i.e. without iterative procedures) the Starink method provides the best accuracy of activation energy analysis methods studied in the literature. It provides an accuracy that is better than $2 \%$ for $E / R T>6$, which covers all known hydrogen desorption reactions, whilst correction for residual errors are possible.
\end{abstract}

Keywords: hydrogen desorption, thermal analysis, thermal desorption spectroscopy, linear heating, activation energy, reaction modelling 


\section{Introduction}

Analysis of the mechanisms of absorption and desorption of hydrogen in materials is important as it improves our understanding of two key areas of technology: solid-state hydrogen storage for cleaner energy applications and hydrogen assisted embrittlement (HAE) in structural materials. In the energy application, hydrogen storage systems are a crucial part to allow a hydrogen economy to work, and material with a high storage capacity are required [1,2]. In structural materials, hydrogen absorption can lead to HAE and premature, unexpected failure of structural components. In both cases, the activation energies of absorption and desorption are particularly important parameters. For hydrogen storage materials a low activation energy is indicative of good applicability of the material for hydrogen storage systems [1]. In studies of hydrogen assisted embrittlement (HAE) in structural materials, determination of the activation energy, $E$, provides information on the trap states of the $\mathrm{H}$ atoms [3].

A regularly employed method for analyses of hydrogen desorption kinetics is linear heating hydrogen desorption measurements. Through performing experiments at several heating rates, $\beta$, the (effective) activation energy of the process can be determined from the shift of a characteristic temperature, $T_{\mathrm{f}}$, of the hydrogen evolution effect with changing heating rate [4,5]. (Often $T_{\mathrm{f}}$ is taken as the temperature of a (local) maximum in $\mathrm{H}$ evolution rate, i.e. a peak temperature $T_{\mathrm{p}}$.) In a range of works (e.g. [5,6,7,8,9,10]) such an activation energy determination has been performed with the aid of an analysis method that is generally known as the Kissinger method, i.e. plots of $\ln \left(T_{\mathrm{p}}{ }^{2} / \beta\right)$ versus $1 / T_{\mathrm{p}}$ are made, and the slope of the straight line is taken to equal $E / R$ ( $R$ is the gas constant). Also the Ozawa method has been regularly employed, see e.g. [11], as well as the Starink method [12]. The thus obtained (apparent) activation energies have been discussed in terms of operating mechanisms and compared with theoretically derived trap energies and chemical bonding states [13]. Materials for hydrogen storage include materials based on chemical storage processes (e.g. in metal hydrides) and by physisorption processes (e.g. in carbon materials: fullerenes, nanotubes, grapheme; zeolites) $[2,14,15]$. Promising new developments to improve hydrogen sorption include application of reactive hydride composites (RHC) [16,17] and the use of nanoconfinement in porous material, e.g. in a (meso)porous medium $[18,19]$. Published desorption data relevant for the present analysis of kinetics includes materials based on $\mathrm{Mg}-\mathrm{Li}$ (e.g. $\left.\mathrm{Mg}\left(\mathrm{NH}_{2}\right)_{2} / \mathrm{LiH} \quad[20,21]\right)$ and $\mathrm{Mg}-\mathrm{Ti} \quad([22,23])$ systems. For structural materials published data on $\mathrm{H}$ desorption includes work on $\mathrm{Al}$ alloys and steels $[24,25,26]$.

Whilst this approach has often appeared to be fruitful, it appears to be generally ignored that all the above mentioned activation energy determination methods have a limited accuracy $[27,28,29,30]$. In this contribution, the accuracy of the activation energy analysis methods for linear heating experiments relevant for $\mathrm{H}$ desorption will be assessed. It will be shown that accuracy of reported activation energies varies, and in several cases reported measured apparent activation energies will be in error by 4 to $6 \%$, and corrections for these deviations are presented. Also, improved methods with higher accuracy are identified.

In addition to the special importance of these activation energy determination methods in the analysis of $\mathrm{H}$ desorption, the methods have been widely applied to a wide range of other reactions. Just as for $\mathrm{H}$ desorption studies the methods are generally applied with little or no reference to their accuracies. In chemistry, the Ozawa (and related Flynn-Wall-Ozawa) method still finds substantial application, even though it has been shown the method is the most inaccurate of its group [27]. A further aim of the present work is thus to further 
clarify the accuracies of the activation energy determination methods.

\section{Derivations of the methods}

\subsection{Model-free methods}

In general it is beneficial to calculate the activation energy using a method that does not depend on the reaction model/mechanism, i.e. a model-free method. The general derivation of model-free activation energy analysis methods has been presented in detail elsewhere [27]. The main elements relevant for hydrogen absorption and desorption studies is summarised in this chapter.

In deriving model-free activation energy analysis methods the transformation rate (e.g. the rate of hydrogen desorption) is considered to be the product of two functions, one depending solely on the temperature, $T$, and the other depending solely on the fraction transformed, $\alpha[27,31]$ :

$$
\frac{d \alpha}{d t}=f(\alpha) k(T)
$$

where $f(\alpha)$ is the transformation function. The temperature dependent function is assumed to follow an Arrhenius type dependency:

$$
k=k_{o} \exp \left(-\frac{E}{R T}\right)
$$

Inserting Eq. (2) into Eq. (1) and taking the logarithm provides

$$
\ln \frac{\mathrm{d} \alpha}{\mathrm{dt}}=-\frac{E}{R T_{f}}-\ln f(\alpha)
$$

To address reactions occurring during heating at a constant heating rate, $\beta$, Eq. (2) is inserted in Eq. (1) and this is integrated by separation of variables:

$$
\int_{0}^{\alpha} \frac{d \alpha}{f(\alpha)}=\frac{k_{o}}{\beta} \int_{0}^{T_{f}} \exp \left(-\frac{E}{R T}\right) d T=\frac{E k_{o}}{R \beta} \int_{y_{f}}^{\infty} \frac{\exp (-y)}{y^{2}} d y
$$

where $y=E / R T, y_{f}=E / R T_{\mathrm{f}}, T_{\mathrm{f}}$ is the temperature at an equivalent (fixed) state of transformation. The integral on the right hand side is generally termed the (Arrhenius) temperature integral, $p(y)$ :

$$
\int_{y_{f}}^{\infty} \frac{\exp (-y)}{y^{2}} d y=p\left(y_{f}\right)
$$

A range of approximations of the temperature integral $\mathrm{p}(y)$ have been suggested in the literature. The asymptotic expansion after a single integration in parts provides:

$$
p(y)=\frac{\exp (-y)}{y^{2}}\left(1+\frac{2 !}{-y}+\frac{3 !}{(-y)^{2}}+\frac{4 !}{(-y)^{3}} \ldots\right)
$$

The first term in the expansion in Eq. 6 is the approximation used by Murray and White [32]:

$$
p(y) \cong \frac{\exp (-y)}{y^{2}}
$$

Doyle [33,34] suggested a linear approximation of the logarithm of $\mathrm{p}(y)$ :

$$
\log p(y) \cong-0.4567 y-2.315
$$

which is equivalent with:

$$
p(y) \cong \exp (-1.0518 y-5.330)
$$

The latter two approximations are part of a wider group of approximations described by:

$$
p(y) \cong \frac{\exp (-A y+B)}{y^{\kappa}}
$$


This class of approximations is important because it leads to a group of methods that is most used (the direct methods) which includes the KissingerAkahira-Sunose (KAS) method [35] (also known as the generalised Kissinger method) and FlynnWall-Ozawa (FWO) [36,37] analysis method. For each value of exponent $\kappa, A$ and $B$ can be optimised by minimising the deviation between the approximation function and the exact integral.

A highly accurate approximation, which outperforms the approximations in Eqs. 7 and 8 over most of the relevant range of $y$ [27], is given by:

$$
p(y) \cong \frac{\exp (-1.0008 y-0.312)}{y^{1.92}}
$$

The above approximations for $\mathrm{p}(y)$ can be used to derive isoconversion methods for activation energy determination. In general all approximations that are of the form of Eq. 10 lead to an isoconversion method with general form $[27,38]$ :

$$
\ln \frac{\beta}{T_{f}{ }^{\kappa}}=-A \frac{E}{R T_{f}}+C_{3}
$$

In Doyle's approximation (Eq. 8) $\kappa=0$ and this provides that $E$ is determined from the slope of plots of $\ln (\beta)$ versus $1 / T_{\mathrm{f}}$. This method is known as the Flynn-Wall-Ozawa (FWO) method and requires multiplying the slope by $R / 1.0518$ [36]. In the approximation in Eq. 7, $\kappa=2$ and this provides that $E$ is determined from the slope of plots of $\ln \left(\beta / T_{\mathrm{f}}{ }^{2}\right)$ versus $1 / T_{\mathrm{f}}[30,35]$. This method is known as the Kissinger-Akahira-Sunose (KAS) method and requires multiplying the slope by $R$. In the approximation in Eq. 11, $\kappa=1.92$ and this provides that $E$ is determined from the slope of plots of $\ln \left(\beta / T_{\mathrm{f}}{ }^{1.92}\right)$ versus $1 / T_{\mathrm{f}}$ and requires multiplying the slope by $R / 1.0008$. This method is often referred to as the Starink method (or Starink
1.92 method) [27]. The main characteristics of these methods are summarised in Table 1.

The deviations introduced in the determination of $E$ in these methods can be determined exactly, and is independent of reaction type [27]. These accuracies are provided in Fig. 1 in terms of the relative deviation $\Delta E / E$ for 3 relevant methods (FWO, KAS, Starink). This figure shows that the methods introduce substantial deviations especially when $y(=E / R T)$ is smaller than 15 , and even the more accurate methods loose accuracy when $y$ drops below 10. Many hydrogen desorption reactions with $y<10$ have been analysed with a range of methods (e.g. Kissinger and Ozawa methods) and hence a range of apparent activation energies reported in the literature will suffer from this inaccuracy.

As the methods described in this section have relative deviation $\Delta E / E$ that are independent of reaction model, they can be considered as genuinely 'model-free' methods, and if required determined activation energies can be corrected for by using Fig. 1, or correction tables such as those presented by Flynn [39].

The above methods all consider temperatures at a constant amount transformed. If it is assumed that the maximum reaction rate (i.e. the peak in a $\mathrm{H}$ evolution or TGA measurement) occurs at a constant amount transformed then we can apply the method at the peak temperatures, $T_{\mathrm{p}}$. For instance, if the approximation of Eq. 7 is applied, $E$ is determined from the slope of plots of $\ln \left(\beta / T_{\mathrm{p}}^{2}\right)$ versus $1 / T_{\mathrm{p}}$. This method is generally termed the Kissinger method, and the approximations and assumption used here are identical to those used in the original work by Kissinger [40,41]. Similarly, one might use approximation Eq. 8, which leads to the method known as the Ozawa (peak) method, or Eq. 10, which leads to the method known as the Starink peak method [27]. However, as we shall see below, these peak methods introduce new uncertainties and are not strictly model-free methods. 
published as International Journal of Hydrogen Energy 43 (2018) pp. 6632-6641

\begin{tabular}{|l|c|c|c|}
\hline \multicolumn{1}{|c|}{ methods } & plot & deviation $\Delta E / E$ & \\
\hline - True isoconversion methods & & at $E / R T=6$ & at $E / R T=15$ \\
\hline Flynn-Wall-Ozawa & $\ln (\beta)$ versus $1 / T_{\mathrm{f}}$ & 0.25 & 0.075 \\
\hline Kissinger-Akahira-Sunose & $\ln \left(\beta / T_{\mathrm{f}}{ }^{2}\right)$ versus $1 / T_{\mathrm{f}}$ & -0.04 & -0.007 \\
\hline Starink 1.92 & $\ln \left(\beta / T_{\mathrm{f}}{ }^{1.92}\right)$ versus $1 / T_{\mathrm{f}}$ & -0.02 & -0.003 \\
\hline - Peak methods & & & \\
\hline Kissinger (= Choo and Lee) & $\ln \left(\beta / T_{\mathrm{p}}{ }^{2}\right)$ versus $1 / T_{\mathrm{p}}$ & -0.04 to +0.2 & -0.01 to 0.011 \\
\hline Starink 1.92 peak & $\ln \left(\beta / T_{\mathrm{p}}{ }^{1.92}\right)$ versus $1 / T_{\mathrm{p}}$ & -0.02 to +0.2 & -0.005 to 0.016 \\
\hline
\end{tabular}

Table 1: Summary of main activation energy analysis methods including the type of plot made and the resulting deviation $(\Delta E / E)$ due to the approximations made.

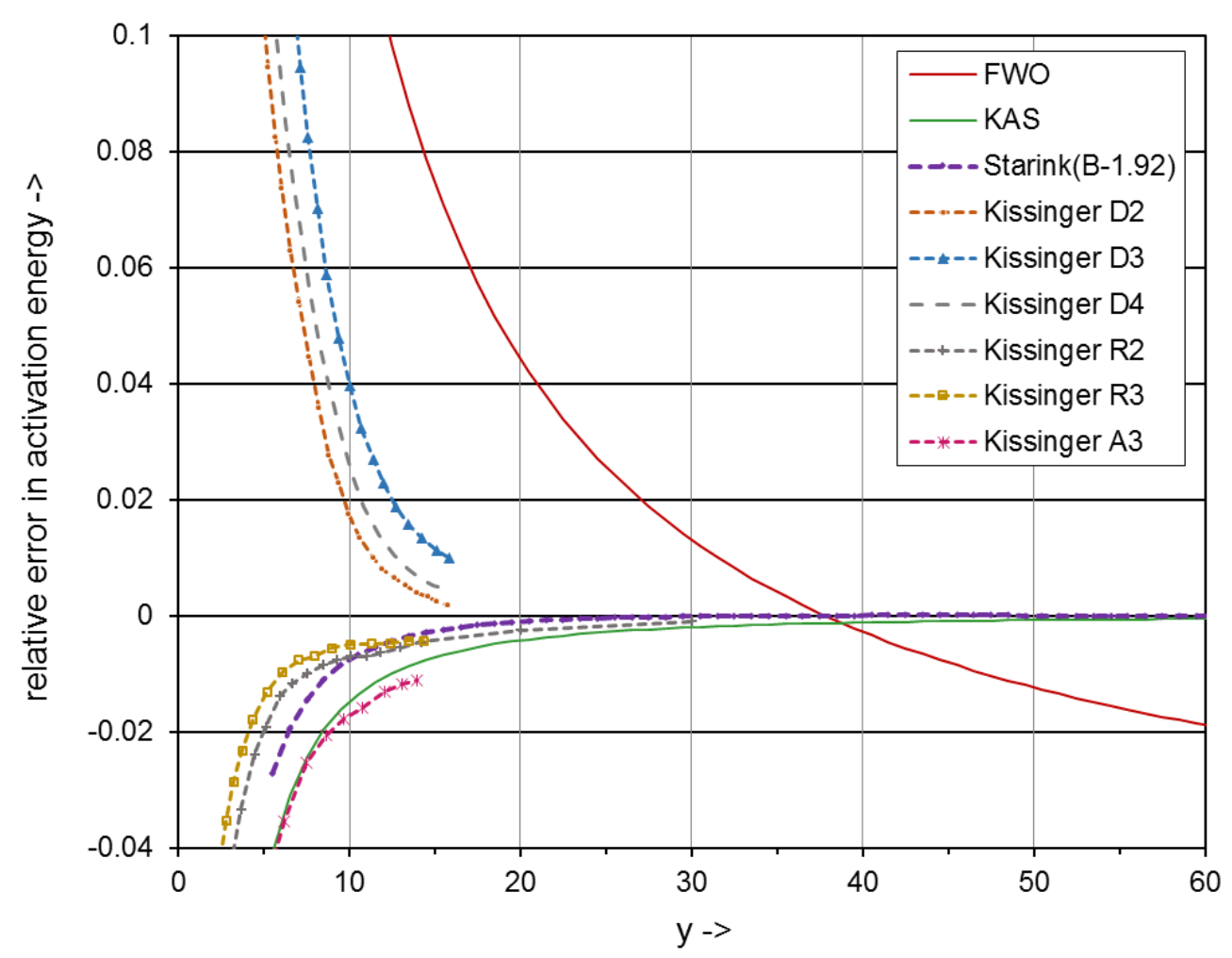

Fig. 1 Relative accuracy of activation energy determination from various methods as a function of $y$ $(=E / R T)($ from $[27,42])$.

FWO = Flynn-Wall-Ozawa isoconversion method, KAS =Kissinger-Akahira-Sunose isoconversion method, Starink $=$ Starink $B=1.92$ isoconversion method [27]. These 3 methods have deviation that is independent of reaction type. Kissinger D2, D3, D4, R2, R3, A3 are the Kissinger peak method applied to the D2, D3, D4, $\mathrm{R} 2, \mathrm{R} 3, \mathrm{~A} 3$ reaction types.

2.2 Peak methods: the original Kissinger method, the 'Choo and Lee method', and other methods.

The above methods (KAS, FWO, Starink 1.92) make no assumption on the transformation model $f(\alpha)$, i.e. they are 'model-free' methods and have been widely employed in analysis of thermally activated reactions in a wide range of fields. However, in the literature on $\mathrm{H}$ desorption the derivation of the peak shift method presented by Choo and Lee [5] has been extensively invoked, 
but that work is little known outside this specific field. In their derivation Choo and Lee assume that the transformation model $f(\alpha)$ conforms to a specific function, e.g. $f(\alpha)$ is proportional to $(1-\alpha)$, and that approach is identical to the approach in Kissinger's 1956 paper [40]. Choo and Lee then proceed to show (as Kissinger did [40]), that the slope of plots of $\ln \left(\beta / T_{\mathrm{p}}^{2}\right)$ versus $1 / T_{\mathrm{p}}$ provides $E / R$. (Such a plot is in various works referred to as a 'Kissinger plot'.) The analysis provided by Choo and Lee [5]* thus does not show the method is a model-free method: the derivation is only valid for one specific type of reaction. In Kissinger's 1957 paper [41] approximations of the temperature integral are employed and this enabled him to show the method is a good approximation to derive the activation energy, providing $y$ is large. The accuracy of the Kissinger method for a wide range of theoretical expressions for reaction models has been analysed by Criado and Ortega [42] and their results for selected models are shown in Fig. 1, for the D2, D3, D4, R2, R3 and A3 models. (For a description of these models, see e.g. $[30,42,43,44]$.$) This figure reveals that the$ accuracy of the Kissinger peak method depends strongly on the reaction model, and the deviations increase with decreasing $y$. Unlike the true modelfree isoconversion methods described in Section 2.2 (e.g. the Starink and KAS methods), the Kissinger peak method (and the identical Choo and Lee peak method) is thus not a fully model-free method, and deviation at low $y$ cannot be corrected for. In general, the Kissinger method overestimates the activation energy for deceleratory reaction models based on diffusion (e.g. the D2 and D3 models which are the 2 and 3 dimensional diffusion models), whilst it causes an underestimate for sigmoid rate equations (e.g. 2 and 3 dimensional Johnson-Mehl-Avrami-

\footnotetext{
* It is noted that the method described by Cho and Lee [5] is thus entirely identical to the method described by Kissinger in his 1965 paper [40]. Rather than referring to this as the 'Cho and Lee method', as occurs in many papers, it should be referred to as the Kissinger (1956) method.
}

Kolmogorov type models, here abbreviated as A2 and A3) and rate equations based on geometric models (e.g. the contraction volume model, R3).

For most reaction models the deviations in the Kissinger peak method are similar in magnitude to that of the KAS method, and the Starink method is (for most models) substantially more accurate than the Kissinger peak method and is always more accurate than the KAS method (see Fig. 1). The Kissinger peak method provides a better accuracy than the Starink method a limited number of reaction models, including the first order reaction and the phase boundary controlled (R3) reaction. It should be noted that investigations of dehydrogenation kinetics in a range of materials indicate the reaction is generally not a first order reaction or a phase boundary controlled reaction $[12,45,47]$. Instead a three-dimensional diffusioncontrolled kinetic mechanism (in Li-Mg-N-H, see [45]) and a Johnson-Mehl-Avrami-Kolmogorov (JMAK) [46] type model (in $\mathrm{Mg}\left(\mathrm{AlH}_{4}\right)_{2}$, see [12]) were identified, whilst in the $\mathrm{Li} 4\left(\mathrm{NH}_{2}\right)_{3} \mathrm{BH}_{4}$ doped $\mathrm{Mg}\left(\mathrm{NH}_{2}\right)_{2}-2 \mathrm{LiH}$ system two controlling mechanisms, one involving 3D diffusion, were found [47]. (However, the progress of dehydrogenation of pure $\mathrm{MgH}_{2}$ has been shown to closely match a first order reaction [48]. Rather than indicating a true first order process, this finding has been interpreted as due to the occurrence of a JMAK [46] nucleation and growth kinetic model with an Avrami coefficient equal to 1, which leads to a kinetic equation identical to a first order reaction equation.) Thus it can be concluded that in general the Kissinger peak method will cause deviations at low $y$. Whilst it may be a convenient method that is often applied ${ }^{\dagger}$, it is not the most accurate method.

\footnotetext{
$\dagger$ In their recent review of analysis methods for hydrogen storage materials (HSM) Pang and Li [4] state that 'almost all the kinetic investigations on the hydrogen storage properties use Kissinger method to calculate the apparent activation energy'. Whilst in a broad sense this is correct, actually both the Kissinger peak method and the Kissinger-Akahira-Sunose method, as well as other methods have been used.
} 


\subsection{Iterative model-free methods with infinite accuracy}

In a full iterative method no graph/slope assessment characteristic of the methods described above is applied, and instead a full computer-based routine is used. Vyazovkin and Dollimore [49] first showed how an accurate and efficient method is derived. To elucidate the main elements of the method, as well as show how it relates to derivations shown above, we will here show the derivation in a slightly modified fashion as follows. Applying Eq. 4 for two heating rates, $\beta_{1}$ and $\beta_{2}$, provides:

$$
\begin{aligned}
& \int_{0}^{\alpha} \frac{d \alpha}{f(\alpha)}=\frac{k_{o}}{\beta_{1}} \int_{T_{S}}^{T_{f 1}} \exp \left(-\frac{E}{R T}\right) d T \\
& =\frac{k_{o} E}{\beta_{1} R} \int_{y_{f 1}}^{y_{S}} \frac{\exp (-y)}{y^{2}} d y=\frac{k_{o} E}{\beta_{2} R} \int_{y_{f 2}}^{y_{S}} \frac{\exp (-y)}{y^{2}} d y
\end{aligned}
$$

where $T_{\mathrm{s}}$ is the start temperature of the heating scan ${ }^{*}$ The latter equation provides:

$$
\beta_{2} \int_{y_{f 1}}^{y_{s}} \frac{\exp (-y)}{y^{2}} d y=\beta_{1} \int_{y_{f 2}}^{y_{s}} \frac{\exp (-y)}{y^{2}} d y
$$

Which is equivalent to:

$$
\begin{aligned}
& \beta_{2}\left\lfloor p\left(y_{f 1}\right)-p\left(y_{S}\right)\right\rfloor \\
& \quad-\beta_{1}\left[p\left(y_{f 2}\right)-p\left(y_{S}\right)\right]=0
\end{aligned}
$$

or

$$
\beta_{2}\left[p\left(\frac{E}{R T_{f 1}}\right)-p\left(\frac{E}{R T_{S}}\right)\right]-\beta_{1}\left[p\left(\frac{E}{R T_{f 2}}\right)-p\left(\frac{E}{R T_{S}}\right)\right]=0
$$

Using a series expansion algorithm the four $p(y)$ terms can be calculated to any required accuracy, and hence in an iteration algorithm $E$ can be devised. One way of enhancing the computational

$\sharp$ For details of the influence of the choice of $T_{\mathrm{s}}$, see Ref. [38], and the Discussion section ( $4^{\text {th }}$ paragraph). efficiency is by using an efficient approximation for $p(y)$ and the Senum and Yang [50] equations clearly provide such a convenient, highly accurate and efficient approximation. Their fourth order approximation is given by ${ }^{\S}$ :

$$
h(y)=\frac{y^{4}+18 y^{3}+86 y^{2}+96 y}{y^{4}+20 y^{3}+120 y^{2}+240 y+120}
$$

As the iteration using Eq. 15 provides $E$ for two heating rates, $\beta_{1}$ and $\beta_{2}$, we will term this $E_{1-2}$. If there are more than two experiments more $E_{\mathrm{i}-\mathrm{j}}$ can be calculated, i.e. if there were 3 heating rates we could obtain $E_{1-2}, E_{1-3} E_{2-3}$. A procedure for averaging these is then needed. (This averaging procedure is the equivalent of the fitting of a straight line through the plot in methods A and B, which can be achieved through a least squares method.) This averaging is conveniently done by minimising the term:

$$
\sum_{i \neq j}^{i} \sum^{j} \beta_{j}\left[p\left(\frac{E}{R T_{f, i}}\right)\right]-\beta_{i}\left[p\left(\frac{E}{R T_{f, j}}\right)\right]=\min
$$

This method was further analysed and popularised in $[29,51]$ and is often referred to as the Vyazovkin method $^{* *}$.

\subsection{Appropriate method for deriving an accurate activation energy}

The above summary of methods shows that if Eq. 1 is valid, a set of data from linear heating experiments with pairs of heating rate and temperature for a fixed state of the reaction can always be analysed to provide an activation energy

\footnotetext{
$\S$ Note that some authors have erroneously quoted the coefficient of $y^{2}$ as 88 and not the correct value of 86 .

** Note that 'Vyazovkin method' has been used by various authors to mean different methods. Also 'Kissinger model/method' and 'Starink model/method' have been used by various authors to mean different activation energy analysis methods.
} 
that is accurate and independent of any approximation on the temperature integral or assumption on the transformation function transformation model $f(\alpha)$. An effective method consists of plotting $\ln \left(\beta / T_{\mathrm{f}}^{1.92}\right)$ versus $1 / T_{\mathrm{f}}$ and multiplying the slope by $R / 1.0008$, with the graph providing a visual assessment of the consistency of the experimental data (i.e. the data should be on a straight line). If particularly high accuracy is required for $y<15$ the obtained $E$ should be corrected using the data in Fig 1.

Alternatively, the Vyazovkin iterative method [51] (see Section 2.3) can be applied but when used to analyse experimental data this method has the drawback that it does not provide a graph which can test the consistency of the experimental data.

\section{Analysis of hydrogen desorption data}

In this section several datasets on hydrogen desorption / dehydrogenation on linear heating from existing literature are reanalysed with a view to either validate or improve the analysis of the activation energies.

\subsection{Hydrogen desorption in metals and alloys for load bearing applications}

We first consider the data on the low temperature $\mathrm{H}$ evolution effect in high purity Fe (with $40 \mathrm{ppm}$ $\mathrm{S}$ and $35 \mathrm{ppm} \mathrm{N}$ ) from the influential work by Choo and Lee [5]. That work presents data on peak temperatures (i.e. the maximum $\mathrm{H}$ evolution rate) for 5 heating rates, which are here reanalysed using the most often used activation energy analysis methods, e.g. the Kissinger peak method, and the FWO and Starink peak methods. The graph of $\ln \left(\beta / T_{\mathrm{p}}{ }^{1.92}\right)$ versus $1 / T_{\mathrm{p}}$, provided in Fig. 2 (blue dots), shows a near perfect straight line, and the slope provides an apparent activation energy of
$17.3 \mathrm{~kJ} / \mathrm{mol}$. The results in Table 2 reveal the different methods provide a range of activation energies $17.0-23.8 \mathrm{~kJ} / \mathrm{mol}$. We also need to consider that as the reaction mechanism is unknown and $E / R T=5.3$ (i.e. very low), the peak methods have a substantial margin of uncertainty (see Fig. 1). For $E / R T=5.3$ the uncertainty amounts to $\sim 10 \%$. Thus the present analysis indicates that even though the value of $17.2 \mathrm{~kJ} / \mathrm{mol}$ reported in [5] is broadly reasonable, it is more accurate and realistic to state that $18 \pm 2 \mathrm{~kJ} / \mathrm{mol}$ is a better reflection of the value with associated uncertainty. (Note that in [5] no analysis of error/uncertainty is provided.) We can also see that use of the Ozawa method would lead to a $\sim 30 \%$ overestimate of the actual activation energy, and this method is thus inappropriate.

Sun et al. [26] provided thermal desorption spectroscopy (TDS) data on the low temperature $\mathrm{H}$ evolution in a $\mathrm{Fe}-0.03 \mathrm{wt} \% \mathrm{C}-16.5 \mathrm{wt} \% \mathrm{Cr}-$ 4.5wt\%Ni-0.9wt\%Mo-0.7wt\%Mn-0.24wt\%Si high strength steel. The data on a 12.9 grade variant of that steel (processed through quenching, cryogenic treatment and tempering, producing a yield strength of $974 \mathrm{MPa}$ ) is provided in Fig. 2 (crosses) and apparent activation energies obtained from the different methods are presented in Table 2. These findings are similar to the findings based on the data for high purity $\mathrm{Fe}$ studied by Choo and Lee [5]: again the activation energy can be determined, but at the low value of $E / R T$ (here it is 4.6) this determination is associated with a relatively large error margin of $\sim 15 \%$. Sun et al. [26] also provides data on a 10.9 grade variant of the same steel and indicated that the activations energy was lower by $1.2 \mathrm{~kJ} / \mathrm{mol}$. However, the present analysis clearly shows that due to the limited accuracy of the method in this range of low $E / R T$ values $( \pm 3$ $\mathrm{kJ} / \mathrm{mol}$ ) a difference cannot be evidenced. 


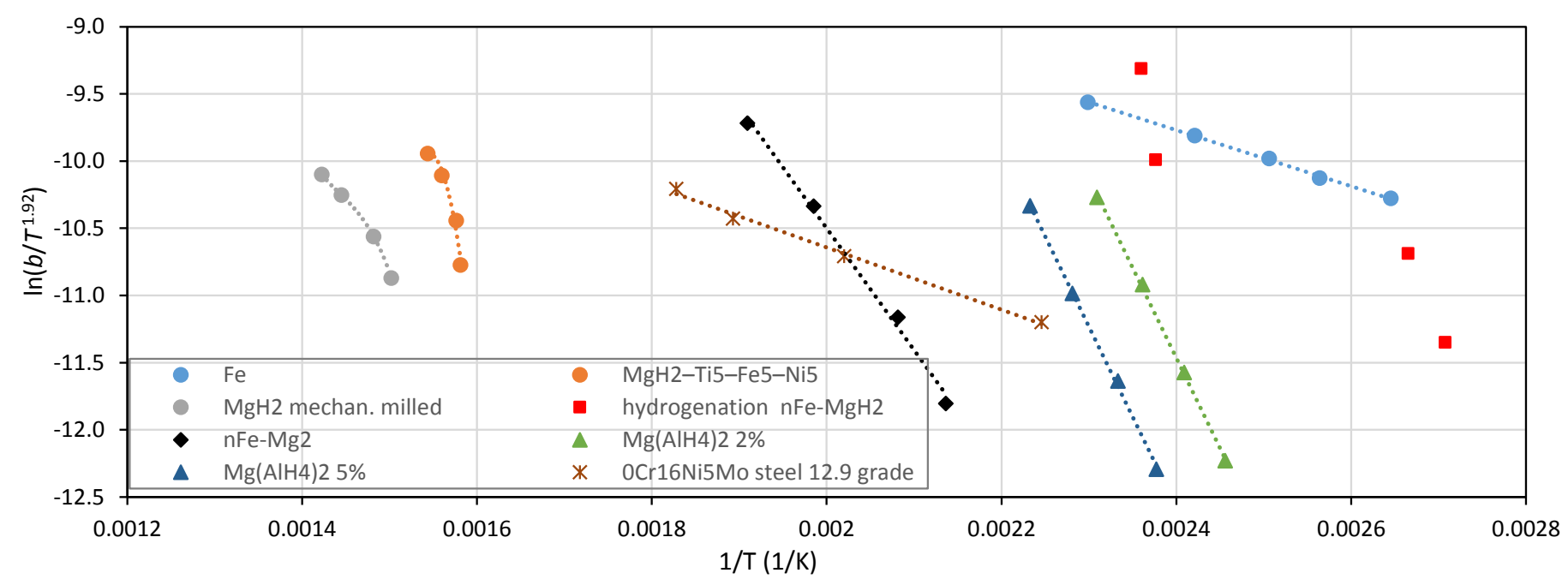

Fig. $2 \ln \left(\beta / T_{\mathrm{f}}{ }^{1.92}\right)$ versus $1 / T_{\mathrm{f}}$ graphs (Starink method) for data from a range of $\mathrm{H}$ desorption experiments and one hydrogenation experiment presented in the literature $[5,12,26,52,53]$. See table 2 for further details of reaction and data sources.

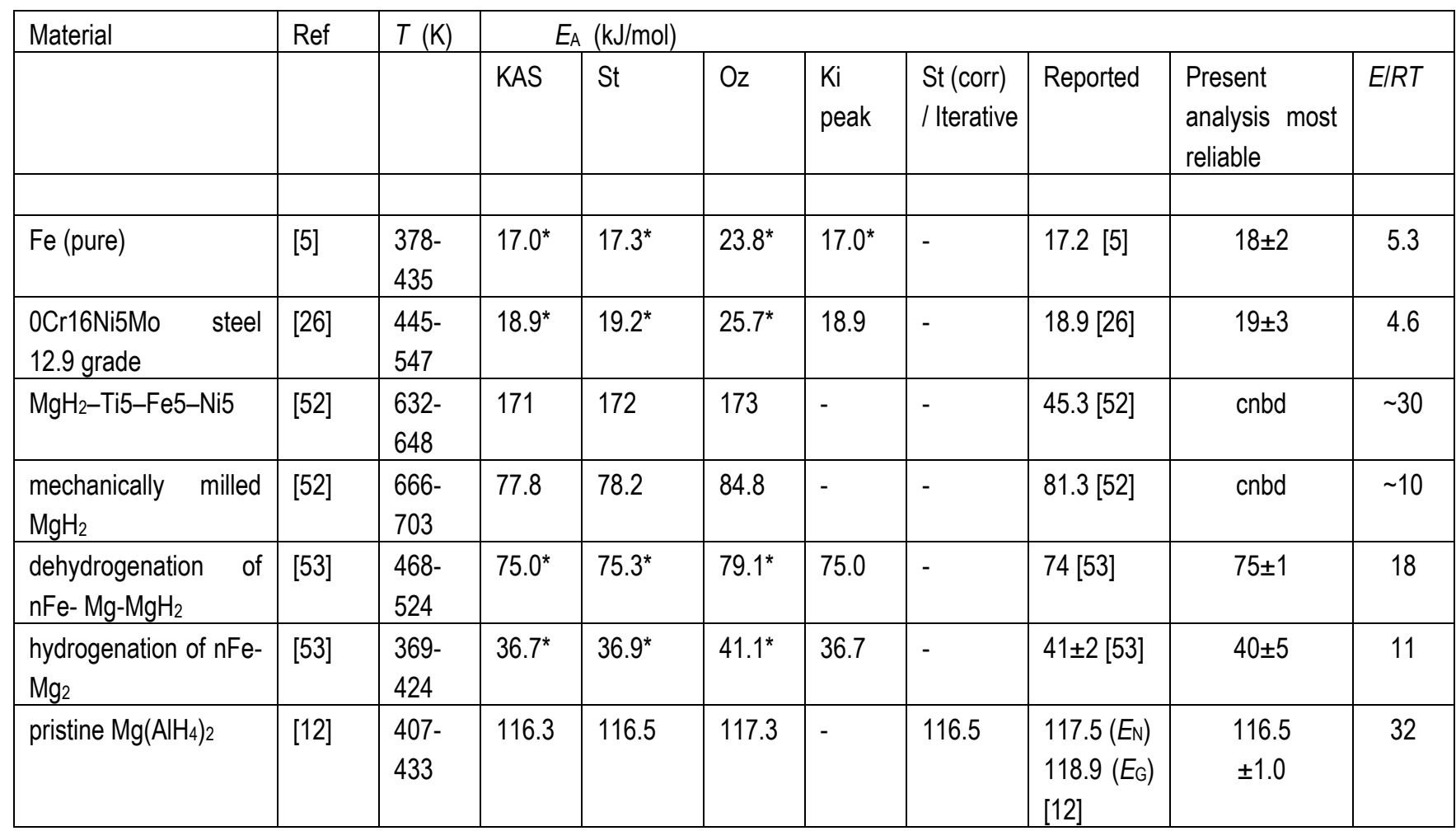

Table 2: Analysis of activation energy for $\mathrm{H}$ evolution from data from a range of literature sources. Reported are activation energies calculated for the present work using the KAS, Starink (St), Ozawa (Oz), Kissinger $(\mathrm{Ki})$ and iterative methods applied to literature data. The column 'reported' refers to activation energies determined in past works in which the $\mathrm{H}$ evolution data was originally reported. The column 'Present analysis most accurate' presents the conclusions from the present analysis with realistic margins of error.

cnbd: can not be determined, *data from maximum reaction rate (peak temperature). 


\subsection{Hydrogen desorption in materials for hydrogen} storage

The data on desorption of from hydrogen storage materials available in the literature mostly focusses on metal hydrides, and thus the present analysis focusses on those materials. Shahi et al. [52] presented data on dehydrogenation of mechanically milled nano-structured $\mathrm{MgH}_{2}$ with additions of metallic elements. Dehydrogenation was measured using a pressure-compositiontemperature (PCT) apparatus. The $\ln \left(\beta / T_{\mathrm{f}}{ }^{1.92}\right)$ versus $1 / T_{\mathrm{f}}$ graphs for $T_{\mathrm{f}}$ at $3 \mathrm{wt} \%$ desorption (which is close to $50 \%$ of completion of the desorption reaction) are presented in Fig. 2. These graphs show that $\ln \left(\beta / T_{\mathrm{f}}{ }^{1.92}\right)$ versus $1 / T_{\mathrm{f}}$ plots of dehydrogenation data on $\mathrm{MgH}_{2}$ and $\mathrm{MgH}_{2}-\mathrm{Ti} 5-$ Fe5-Ni5 is not on a straight line, with the slope change between the lower and higher heating rate range amounting to a factor of about $3\left(\mathrm{MgH}_{2}\right)$ and $7\left(\mathrm{MgH}_{2}-\mathrm{Ti5}-\mathrm{Fe} 5-\mathrm{Ni5}\right)$ change in slope. (Other plots described in Section 2 provide similar curved plots.) Clearly we cannot derive an activation energy from this data because i) if the data is accurate then the requirement of a single thermally activated reaction is violated and the method is invalid, or ii) if there is (in reasonable approximation) a single thermally activated reaction underlying the measured data then the measured data does not reflect that (e.g. the data or measurement method is inaccurate). In any case, the suggestion (in [52]) that an (apparent) activation energy can be obtained from this data appears incorrect.

Kumar et al. [53] presented data on dehydrogenation of nanometric iron $(\mathrm{nFe})$ doped $\mathrm{Mg}-\mathrm{MgH}_{2}$. The dehydrogenation kinetics during linear heating was analysed using a thermoanalyser unit (TG-DTA) with a mass spectrometer attached to it. The peak temperatures for the desorption (i.e. at the maximum desorption rate) were obtained from the data in [53] and the $\ln \left(\beta / T_{\mathrm{p}}{ }^{1.92}\right)$ versus $1 / T_{\mathrm{p}}$ graph is plotted in Fig. 2 (black diamonds). This graph shows a good linear correlation and hence the reaction and data accuracy should be suitable for derivation of an activation energy. In line with the analysis in [30], the data in Table 2 shows that for this reaction with $E / R T \approx 32$ the Kissinger and Starink methods provide consistent results. We can thus conclude that the activation energy can be derived with good accuracy as $75 \pm 1 \mathrm{~kJ} / \mathrm{mol}$.

Pan and $\mathrm{Li}$ [12] provided data on the first-step dehydrogenation of $\mathrm{Mg}\left(\mathrm{AlH}_{4}\right)$. Here their data for constant heating is reanalysed and data for a $2 \mathrm{wt} \%$ and $5 \mathrm{wt} \%$ dehydrogenation at 4 heating rates is presented in Fig. 2 as $\ln \left(\beta / T_{\mathrm{f}}{ }^{1.92}\right)$ versus $1 / T_{\mathrm{f}}$ plots (triangles). These data show very good linear correlations with slopes indicating activation energies of $116.5 \mathrm{~kJ} /$ mole. As $E / R T \approx 32$ the analysis in Fig. 1 indicates that the Kissinger and Starink methods should provide consistent results, and that is confirmed by the present analysis (Table 2). (It is interesting to note that in their analysis Pan and $\mathrm{Li}$ [12] suggest that the dehydration is diffusion controlled and that curves can be fitted well by the John-Mehl-AvramiKolmogorov model, whilst recent analysis [54] has shown that the JMAK model is not valid for diffusion-controlled reactions - it is at best a reasonable approximation for low fraction transformed [55,56,57]. Thus this issue requires further research.)

In addition the literature contains reports on analyses of linear heating data for which the original data has not been published and hence cannot be reanalysed. Some of this work concerns application of methods where substantial deviations are expected. For instance analysis of the first step of dehydrogenation of ball-milled $\mathrm{NaAlH}_{4}+3 \mathrm{~mol} \% \mathrm{NiFe}_{2} \mathrm{O}_{4}$ by Huang et al. [7] using the Kissinger peak method shows $E=54.3 \mathrm{~kJ} / \mathrm{mol}$ with $E / R T=15$. From Fig. 1 we can see that deviations of about $1 \%$ can be expected. Choi et al. [22] applied an FWO type analysis to desorption analysis in as-milled $4 \mathrm{MgH}_{2} / \mathrm{TiH}_{2}$ for which $E \approx 68 \mathrm{~kJ} / \mathrm{mol}$ with $E / R T \approx 16$. Fig. 1 shows 
that this assessment of $E$ should have a deviation of about $6 \%$ i.e. the actual value should be closer to $E \approx 64 \mathrm{~kJ} / \mathrm{mol}$.

A further issue causing inaccuracies in some works on hydrogen desorption is the application of the FWO (or Ozawa) method, i.e. deriving activation energies from plots of $\ln (\beta)$ versus $1 / T_{\mathrm{f}}$. As shown in Fig. 1 and [27], application of this method can produce deviations between $+20 \%$ and $-2 \%$ over the range of $E / R T$ encountered for $\mathrm{H}$ desorption reactions. For the present analysis a few of the works that apply the FWO method have been reanalysed. In [13] hydrogen uptake and release in mixtures of magnesium hydride $\left(\mathrm{MgH}_{2}\right.$, $98 \%)$ and titanium hydride $\left(\mathrm{TiH}_{2}, 99 \%\right)$ that were processed using high-energy-high-pressure (HEHP) mechanical ball-milling, were analysed. From reanalysing thermogravimetric analysis data in [13] it is found that the activation energies of dehydrogenation of as-milled $7 \mathrm{MgH}_{2} / \mathrm{TiH}_{2}$, asmilled $4 \mathrm{MgH}_{2} / \mathrm{TiH}_{2}$, as-milled $\mathrm{MgH}_{2}$, and asreceived (not milled) $\mathrm{MgH}_{2}$ are 66, 63, 92 and 150 $\mathrm{kJ} / \mathrm{mol}$, respectively. These corrected values are up to $8 \%$ lower than the values given in [13]. This clearly shows that the FWO method is inaccurate and should be avoided, or, if used, should be subject to a correction as described in [27,39]. There are several other reports in the literature where the FWO method is applied to hydrogen desorption data.

In addition to above data, also some data on hydrogen desorption in reactive hydride composites (RHCs) has been published. The data generally involves a limited set of heating rates. It is relevant to note that DSC analysis of $\mathrm{CaF}_{2}$ doped $\mathrm{CaH}_{2} / \mathrm{MgB}_{2}$ RHC shows peak temperatures that provide straight lines on Kissinger (or Starink) plots for 3 consecutive dehydrogenation stages [58]. The slopes provide activation energies $162 \pm 15 \mathrm{~kJ} / \mathrm{mol}, 132 \pm 5 \mathrm{~kJ} / \mathrm{mol}$ and $116 \pm 5 \mathrm{~kJ} / \mathrm{mol}$. The $E / R T$ values are the range 24 to 30 , and thus, as Fig. 1 shows, any uncertainty introduced due to approximations in the Kissinger and Starink analysis methods will be virtually negligible. The uncertainty/error is thus solely due to experimental uncertainties which in this case are substantial. Some activation energy analysis on hydrogen desorption of nanoconfined hydrogen storage material has been published. For instance, analysis of the peak temperature in DSC data revealed that nanoconfinement of $\mathrm{LiBH}_{4}$ and $\mathrm{MgH}_{2}$ in a carbon aerogel scaffold caused a significant reduction of the activation energy of hydrogen desorption to $125 \mathrm{~kJ} / \mathrm{mol}$ [59]. With $E / R T \sim 24$, Fig. 1 shows any uncertainty introduced due to approximations in the Kissinger and Starink analysis methods will be less than $1 \%$.

\section{Discussion}

From the above analysis of literature data, which is summarised in Table 2 and Fig. 2, we can see that the accuracy of the analyses varies over a wide range, from data that can be analysed to provide an activation energy with high accuracy, to data that is clearly unsuitable for analysis. The present reanalysis (summarized in Table 2) provides both examples of more accurate $E_{\mathrm{A}}$ values being derived, as well as changed uncertainty margins due to a more realistic assessment of uncertainties in analysis methods and inconsistency of data with assumptions in the analysis methods. If data is accurate and the correct analysis method is chosen accuracies of better than $1 \%$ can be achieved, whilst poor data in combination with inappropriate application of an analysis method has produced analyses in the literature that are very inaccurate. Thus caution is needed in interpreting activation energy data obtained from linear heating experiments, and accuracy can be assessed based on 2 criteria. Firstly, the accuracy of the method should be considered by comparing accuracy for the $E / R T$ value of the reaction using Fig. 1. Secondly, the linearity of the $\ln \left(\beta / T_{\mathrm{f}}{ }^{1.92}\right)$ versus $1 / T_{\mathrm{f}}$ graph should be carefully considered, as a curved line can be indicative of a reaction that does not follow 
a single activation energy kinetics, or be indicative of inaccurate data.

In relation to the accuracy and uncertainties, it is particularly noted that the data for pristine $\mathrm{Mg}\left(\mathrm{AlH}_{4}\right)_{2}$ in Fig. 2 fits almost perfectly the straight line: correlation coefficients are 0.9996 and 0.9994 for 2 and $5 \%$ mass gain, respectively. In combination with the availability of a highly accurate analysis method, this results in a very low uncertainty to the determined activation energy being assigned. This is quite remarkable; apparently the $\mathrm{H}$ evolution kinetics in these pristine $\mathrm{Mg}\left(\mathrm{AlH}_{4}\right)_{2}$ samples is highly reproducible and the measurements (reported in [12]) were accurate. The remaining uncertainty (estimated at $1 \mathrm{~kJ} / \mathrm{mol}$ ) is due to small deviations from linear correlation.

The activation energy method has also been applied to data from a modelled reaction [7], and this provides a further means of assessing the present analysis. In an analysis of hydrogen diffusion and trapping interactions in pure $\mathrm{Fe}$ Hurley et al. [7] write 'the assigned detrapping activation energy for Type A charging was determined to be $53.4 \mathrm{~kJ} \mathrm{~mol}^{-1}$, which is very close to the imposed $E_{\mathrm{p}}$ value of $53.69 \mathrm{~kJ} \mathrm{~mol}^{-1}$.' With the present analysis of errors induced by the method (Fig. 1) we can now correct the measured value to be $53.7 \mathrm{~kJ} \mathrm{~mol}^{-1}$, which is identical to the imposed $E_{\mathrm{p}}$ value of $53.69 \mathrm{~kJ} \mathrm{~mol}^{-1}$. In fact, it can now be seen that the analysis of activation energy for this Type A simply confirms the present analysis of deviations in the methods.

A further limitation of the accuracy can occur in the (rare) case that the low temperature end of the temperature integral cannot be neglected. This has been analysed in [38], and it shows that deviations occur typically when the product of $E_{\mathrm{A}}$ and the difference between start temperature of the linear heating scan $\left(T_{\mathrm{o}}\right)$ and $T_{\mathrm{f}}$ is small (typically when $\left.\left(T_{\mathrm{o}}-T_{\mathrm{f}}\right) \times E_{\mathrm{A}}<100 \times 100 \mathrm{~K} \cdot \mathrm{kJ} / \mathrm{mol}\right)$. Applying the analysis in [38] to the hydrogen evolution assessed in the present work shows that generally this is not an issue for hydrogen evolution data. A specific example is hydrogen evolution in steels studied with $T_{\mathrm{o}}$ at ambient temperature as in [60]. With $E_{\mathrm{A}}$ typically 20 to $30 \mathrm{~kJ} / \mathrm{mol}$ and $\left(T_{\mathrm{o}^{-}} T_{\mathrm{f}}\right)$ typically $100^{\circ} \mathrm{C}$, deviations due to low temperature end of the temperature integral can amount to several percent. Analysis of hydrogenation reactions can also be prone this type of potential deviation. For instance, the hydrogenation of $\mathrm{nFe}-\mathrm{Mg}_{2}$ was studied in [53] with scans starting at $40^{\circ} \mathrm{C}$, with $\left(T_{\mathrm{o}}-T_{\mathrm{f}}\right) \times E_{\mathrm{A}}$ typically $\sim 80 \times 40 \mathrm{~K} \cdot \mathrm{kJ} / \mathrm{mol}$. In view of this the accuracy of the activation energy determination is limited, as indicated in Table 2.

Finally it is relevant to note that apart from using correct and accurate methods to analyse linear heating experiments, improved reliability of analysis of kinetics can be achieved by performing isothermal experiments at multiple relevant temperatures. (Such experiments will be more time consuming than linear heating experiments [30].)

\section{Conclusions}

The accuracy of available methods for determination of activation energy from linear heating experiments has been analysed and the findings are compared and contrasted to data and analyses of hydrogen evolution in potential hydrogen storage materials and structural materials that are prone to hydrogen embrittlement. It is found that the accuracy of analyses of activation energies reported in the literature for hydrogen evolution varies wildly, with some methods being applied in conditions where the method is inaccurate. Particular findings include:

Due to hydrogen evolution often occurring for low $E / R T$ values, activation energy analysis methods applied to linear heating experiments can produce large errors. Several cases of errors larger than 5\% have appeared in the published literature. 
The Ozawa and Flynn-Wall-Ozawa methods are highly inaccurate and particularly for hydrogen evolution where $E / R T$ is often smaller than 15 they are inappropriate, unless an iterative correction procedure is applied.

The Kissinger peak method is accurate for first order reactions, but as hydrogen evolution reactions generally are not first order reactions, application of this method will result in inaccuracies which increase for decreasing $E / R T$. In general the magnitude of the deviations of the Kissinger peak method are not predictable, as this depends on the reaction model. In many cases the Kissinger peak method is inappropriate for high accuracy determination of activation energy for hydrogen evolution reactions.

Amongst all the methods that provide an activation energy directly from a slope (i.e. without iterative procedures) the Starink method provides the best accuracy of activation energy analysis methods. Particularly for hydrogen evolution reactions in which $E / R T$ is often smaller than 15, and sometimes smaller than 10, this method provides accuracies that are significantly more accurate than other methods applied in the literature. In $\sim 90 \%$ of the hydrogen evolution reactions studied in the literature the Starink method provides an accuracy that is better than $2 \%$. Correction for residual errors are possible.

\section{References}

1 Sadhasivam T, Kim H-T, Jung S, Roh S-H, Park $\mathrm{J}-\mathrm{H}$, Jung $\mathrm{H}-\mathrm{Y}$. Dimensional effects of nanostructured $\mathrm{Mg} / \mathrm{MgH}_{2}$ for hydrogen storage applications: A review. Renew Sust Energy Rev 2017, 72, 523-34.

2 Ren J, Musyoka NM, Langmi HW, Mathe M, Liao S. Current research trends and perspectives on materials-based hydrogen storage solutions: A critical review. Int J Hydr Energy 2017, 42, 289311

3 Silverstein R, Eliezer D, Glam B, Eliezer S, Moreno D. Evaluation of hydrogen trapping mechanisms during performance of different hydrogen fugacity in a lean duplex stainless steel. J All Comp 2015, 648, 601-8
4 Pang Y, Li Q. A review on kinetic models and corresponding analysis methods for hydrogen storage materials. Inter J Hydr Energy 2016, 41, 18072-87.

5 Choo W, Lee JY. Thermal analysis of trapped hydrogen in pure iron. J Mater Sci 1982; 17:1930e8.

6 Zhang T, Isobe S, Jain A, Wang Y, Yamaguchi S, Miyaoka H, Ichikawa T, Kojima Y, Hashimoto N. Enhancement of hydrogen desorption kinetics in magnesium hydride by doping with lithium metatitanate. J All Comp 2017, 711, 400-5.

7 Huang Y, Li P, Wan Q, Zhang J, Li Y, Li R, Dong $\mathrm{X}, \mathrm{Qu} \mathrm{X}$. Improved dehydrogenation performance of $\mathrm{NaAlH} 4$ using $\mathrm{NiFe} 2 \mathrm{O} 4$ nanoparticles. J All Comp 2017, 709, 850-6.

8 Hurley C, Martin F, Marchetti L, Chêne J, Blanc C, Andrieu E. Numerical modeling of thermal desorption mass spectroscopy (TDS) for the study of hydrogen diffusion and trapping interactions in metals. Inter J Hydr Energy 2015,40, 3402-14.

9 Révész Á, Gajdics M, Schafler E, Calizzi M, Pasquini L. Dehydrogenation-hydrogenation characteristics of nanocrystalline $\mathrm{Mg} 2 \mathrm{Ni}$ powders compacted by high-pressure torsion. J All Comp 2017, 702, 84-91.

10 Kim SJ, Ryu KM, Oh MS. Addition of cerium and yttrium to ferritic steel weld metal to improve hydrogen trapping efficiency. Inter J Min Metall Mater 2017, 24, 415-22.

11 Markmaitree T, Osborn W, Shaw LL. Comparisons between $\mathrm{MgH} 2$ - and $\mathrm{LiH}$-containing systems for hydrogen storage applications. Inter $\mathbf{J}$ Hydr Energy 2008, 33, 3915-24.

12 Pang Y, Li Q. Insight into the kinetic mechanism of the first-step dehydrogenation of $\mathrm{Mg}\left(\mathrm{AlH}_{4}\right)_{2}$. Scr Mater 2017, 130, 223-8.

13 Jung JH, Kim D, Hwang J, Lee YL. Theoretical study on the hydrogen storage mechanism of the $\mathrm{Li}-\mathrm{Mg}-\mathrm{N}-\mathrm{H}$ system. Ihm J, Inter J Hydr Energy 2016, 41, 17506-17510.

14 Niaz S, Manzoor T, Pandith AH. Hydrogen storage: Materials, methods and perspectives, Renewable Sust Energy Rev. 2015, 50, 457-69

15 Durbin DJ, Malardier-Jugroot C. Review of hydrogen storage techniques for on board vehicle applications. Inter J Hydr Energy 2013, 38, 14595-14617.

16 Jepsen LH, Ley MB, Lee YS, Cho YW, Dornheim M, Jensen JO, Filinchuk Y, Jørgensen JE, Besenbacher F, Jensen TR. Boron-nitrogen based hydrides and reactive composites for hydrogen storage. Mater Today 2014, 17, 129-35. 
17 Sulaiman NN, Ismail M. Study the effect of $\mathrm{SrFe} 12 \mathrm{O} 19$ on $\mathrm{MgH}_{2} / \mathrm{LiAlH}_{4}$ composite for solidstate hydrogen storage. Hydr Energy 2017, 42, 29830-39.

18 Ngene P, van Zwienen, Jongh PE. Reversibility of the hydrogen desorption from $\mathrm{LiBH}_{4}$ : a synergetic effect of nanoconfinement and $\mathrm{Ni}$ addition. Chem Commun 2010, 46 8201-03

19 Li Y, Zhou G, Fang F, Yu X, Zhang Q, Ouyang L, Zhu M, Sun D. De-/re-hydrogenation features of $\mathrm{NaAlH}_{4}$ confined exclusively in nanopores. Acta Mater 2011, 59, 1829-38

20 Shahi R, Raghubanshi H, Shaz MA, Srivastava ON, Inter J Hydr Energy 2013, 38, 8863-71.

21 Yap FAH, Ismail $M$. The hydrogen storage properties of $\mathrm{Mg}$-Li-Al composite system catalyzed by $\mathrm{K}_{2} \mathrm{ZrF}_{6}$. J Phys Chem Sol 2017, 104, 214-20.

22 Choi YJ, Lu J, Sohn HY, Fang ZZ. Hydrogen storage properties of the Mg-Ti-H system prepared by high-energy-high-pressure reactive milling. J Power Sources 2008, 180, 491-97.

23 Cheng H, Chen Y, Sun W, Lou H, Liu Y, Qi Q, Zhang J, Liu J, Yan K, Jin H, Zhang Y, Yang S. The enhanced dehydrogenation performances of $17 \mathrm{MgH}_{2}-12 \mathrm{Al}$ composite with additive $\mathrm{TiH}_{2}$. J All Comp 2017, 704, 769-775.

24 Legrand E, Oudriss A, Savall C, Bouhattate J, Feaugas X, Int J Hydr Energy 2015, 40, 28712881.

25 Beidokhti B, He P, Kokabi AH, Dolati A. Control of hydrogen cracking in the welded steel using microstructural traps. Mater Sci Techn 2017, 33, 408-14.

26 Sun YW, Chen JZ, Liu J. Investigation into Hydrogen Diffusion and Susceptibility of Hydrogen Embrittlement of High Strength 0Cr16Ni5Mo Steel. J Iron Steel Res Intern 2015, 22, 961-68.

27 Starink M. The Determination of Activation Energy from Linear Heating Rate Experiments: a Comparison of the Accuracy of Isoconversion Methods. Thermochim Acta 2003, 404, 163-176

28 Shissler DJ, Fredrick SJ, Braun MB, Pieto AL. Magnesium and Doped Magnesium Nanostructured Materials for Hydrogen Storage. In 'Low-cost Nanomaterials: Toward Greener and More Efficient Energy Applications', Lin Z, Wang J, editors, London: Springer, 2014, p. 297320.

29 Vyazovkin S. Isoconversional Kinetics of Thermally Stimulated Processes; Switzerland, Springer International Publishing; 2015.
30 Starink MJ. The analysis of Al-based alloys by calorimetry: quantitative analysis of reactions and reaction kinetics. Intern Mater Rev 2004, 49, 191226.

31 Gao X, Chen D and Dollimore D. The correlation between the value of $\alpha$ at the maximum reaction rate and the reaction mechanisms: A theoretical study. Thermochim Acta 1993, 223, 75-82

32 Murray $P$, White J. Kinetics of the thermal decomposition of clay; Part 4: Interpretation of DTA to thermal analysis of clays. Trans Brit Ceram Soc 1955, 54, 204-37

33 Doyle CD. Kinetic analysis of thermogravimetric data. J Appl Polym Sci 1961, 5, 285-92

34 Doyle CD. Series Approximations to the Equation of Thermogravimetric Data. Nature 1965, 207, 290-91

35 Akahira T, Sunose T. Transactions joint convention of four electrical institutes, paper no. 246, Chiba Institute of Technology, J Sci Educ Techn 1971, 16, 22-31.

36 Flynn JH, Wall LA. A quick, direct method for the determination of activation energy from thermogravimetric data. J Polym Sci Part B 1966, 4, 323-26

37 Ozawa T. Estimation of activation energy by isoconversion methods. Thermochim Acta 1992, 203, 159-65

38 Starink MJ. Activation energy determination for linear heating experiments: deviations due to the low temperature end of the temperature integral. J Mater Sci 2007, 42, 483-89.

39 Flynn JH, The isoconversional method for determination of energy of activation at constant heating rates: Corrections for the Doyle approximation. J Thermal Anal 1983, 27, 95-102

40 Kissinger HE. Variation of peak temperature with heating rate in differential thermal analysis. J Res Nat Bur Stand 1956, 57, 217-21

41 Kissinger HE. Reaction Kinetics in Differential Thermal Analysis. Analyt Chem 1957, 29, 1702

42 Criado JM, Ortega A. Non-isothermal transformation kinetics: remarks on the Kissinger method. J Non Cryst Solids 1986, 87, 302-11.

43 Pérez-Maqueda LA, Criado JM, Sánchez-Jiménez PE. Combined Kinetic Analysis of Solid-State Reactions: A Powerful Tool for the Simultaneous Determination of Kinetic Parameters and the Kinetic Model without Previous Assumptions on the Reaction Mechanism. J Phys Chem A 2006, 110, 12456-62.

44 Galwey AK, Brown ME. Kinetic Background to Thermal Analysis and Calorimetry. In: Brown ME, Gallagher, editors, Handbook of Thermal 
Analysis and Calorimetry, Volume 1, Amsterdam: Elsevier; 1998, p. 147-224.

45 Liu Y, Zhong K, Luo K, Gao M, Pan H, Wang Q. Size-Dependent Kinetic Enhancement in Hydrogen Absorption and Desorption of the Li-Mg-N-H System. J Am Chem Soc 2009, 131, $1862-70$.

46 Starink MJ. On the meaning of the impingement parameter in kinetic equations for nucleation and growth reactions. J Mater Sci, 2001, 36: 4433-41.

47 Amica G, Cova F, Arneodo Larochette P, Gennari FC. Two-controlling mechanisms model for hydrogen desorption in the $\mathrm{Li}_{4}\left(\mathrm{NH}_{2}\right)_{3} \mathrm{BH}_{4}$ doped $\mathrm{Mg}\left(\mathrm{NH}_{2}\right)_{2}-2 \mathrm{LiH}$ system. Inter $\mathbf{J} \mathrm{Hydr}$ Energy 2017, 42, 6127-36.

48 Perejón A, Sánchez-Jiménez PE, Criado J.M, Pérez-Maqueda LA. Magnesium hydride for energy storage applications: The kinetics of dehydrogenation under different working conditions J All Comp 2016, 681, 571-79.

49 Vyazovkin S, Dollimore D. Linear and Nonlinear Procedures in Isoconversional Computations of the Activation Energy of Nonisothermal Reactions in Solids. J Chem Inf Comp Sci 1996, $36,42-45$

50 Senum GI, Yang RT. Rational approximations of the integral of the Arrhenius function. J Thermal Anal 1977, 16, 445-47

51 Vyazovkin S, Evaluation of activation energy of thermally stimulated solid-state reactions under arbitrary variation of temperature. J Comput Chem, 1997, 18, 393-402

52 Shahi RR, Tiwari AP, Shaz MA, Srivastava ON. Studies on de/rehydrogenation characteristics of nanocrystalline $\mathrm{MgH}_{2}$ co-catalyzed with $\mathrm{Ti}, \mathrm{Fe}$ and Ni. Inter J Hydr Energy 2013, 38, 2778-84.

53 Kumar S, Singh A, Tiwari AG, Kojima Y, Kain $\mathrm{V}$. Thermodynamics and kinetics of nanoengineered $\mathrm{Mg}-\mathrm{MgH}_{2}$ system for reversible hydrogen storage application Thermochim Acta 2017, 652, 103-108.

54 Starink MJ. A new model for diffusion-controlled precipitation reactions using the extended volume concept. Thermochim Acta 2014, 596, 109-19.

55 Starink MJ. Analysis of nucleation and growth with the model for diffusion-controlled precipitation reactions based on the extended volume concept. J All Comp 2015, 630, 250-255.

56 Starink MJ. Kinetic equations for diffusioncontrolled precipitation reactions. J Mater Sci 1997, 32 4061-70.

57 Starink MJ, Milkereit B, Zhang Y, Rometsch PA. Predicting the quench sensitivity of $\mathrm{Al}-\mathrm{Zn}-\mathrm{Mg}-\mathrm{Cu}$ alloys: a model for linear cooling and strengthening. Mater Design 2015, 88, 958-71.

58 Suarez Alcantara K, Boesenberg U, Zavorotynska O, Bellosta von Colbe J, Taube K, Baricco M, Klassen T, Dornheim M. Sorption and desorption properties of a $\mathrm{CaH}_{2} / \mathrm{MgB}_{2} / \mathrm{CaF}_{2}$ reactive hydride composite as potential hydrogen storage material, J Sol St Chem, 184, 2011, 3104-9.

59 Gosalawit-Utke R, Thiangviriya S, Javadian P, Laipple D, Pistidda C, Bergemann N, Horstmann C, Jensen TR, Klassen T, Dornheim M. Effective nanoconfinement of $2 \mathrm{LiBH}_{4}-\mathrm{MgH}_{2}$ via simply $\mathrm{MgH}_{2}$ premilling for reversible hydrogen storages. Int J Hydr Energy 2014, 39, 15614-26

60 Asahi H, Hirakami D, Yamasaki S. Hydrogen Trapping Behavior in Vanadium-added Steel. ISIJ Intern 2003, 43, 527-33. 